\title{
On the existence of a symplectic desingularization of some moduli spaces of sheaves on a K3 surface
}

\author{
Young-Hoon Kiem
}

\begin{abstract}
Let $M_{c}$ be the moduli space of semistable torsion-free sheaves of rank 2 with Chern classes $c_{1}=0$ and $c_{2}=c$ over a K3 surface with generic polarization. When $c=2 n \geqslant 4$ is even, $M_{c}$ is a singular projective variety which admits a symplectic form, called the Mukai form, on the smooth part. A natural question raised by O'Grady asks if there exists a desingularization on which the Mukai form extends everywhere nondegenerately. In this paper we show that such a desingularization does not exist for many even integers $c$ by computing the stringy Euler numbers.
\end{abstract}

\section{Introduction}

Let $X$ be a projective K3 surface with generic polarization $\mathcal{O}_{X}(1)$ and let $M_{c}=M(2,0, c)$ be the moduli space of semistable torsion-free sheaves on $X$ of rank 2, with Chern classes $c_{1}=0$ and $c_{2}=c$. When $c=2 n \geqslant 4$ is even, $M_{c}$ is a singular projective variety. Recently O'Grady raised the following question $[\mathrm{Ogr} 99,(0.1)]$.

Question 1.1. Does there exist a symplectic desingularization of $M_{2 n}$ ?

In [Ogr99], he analyzed Kirwan's desingularization $\widehat{M}_{c}$ of $M_{c}$ and proved that $\widehat{M}_{c}$ can be blown down twice and that as a result he obtained a symplectic desingularization $\widetilde{M}_{c}$ of $M_{c}$ in the case when $c=4$. This turns out to be a new irreducible symplectic variety.

When $c \geqslant 6$, O'Grady conjectures that there is no smooth symplectic model of $M_{c}$ (see [Ogr99, p. 50]). The purpose of this paper is to provide a partial answer to Question 1.1.

Theorem 1.2. There is no symplectic desingularization of $M_{2 n}$ if $n a_{n} /(2 n-3)$ is not an integer where $a_{n}$ is the Euler number of the Hilbert scheme $X^{[n]}$ of $n$ points in $X$.

It is well known that $a_{n}$ is given by the equation

$$
\sum_{n=0}^{\infty} a_{n} q^{n}=\prod_{n=1}^{\infty} 1 /\left(1-q^{n}\right)^{24} .
$$

By direct computation, one can check that $n a_{n} /(2 n-3)$ is not an integer for $n=5,6,8,11,12$, $13,15,16,17,18,19,20, \ldots$.

The idea of the proof is to use properties of the stringy Euler numbers. If there is an irreducible symplectic desingularization $\widetilde{M}_{c}$ of $M_{c}$, then the stringy Euler number of $M_{c}$ is equal to the ordinary Euler number of $\widetilde{M}_{c}$ because the canonical divisors of both $\widetilde{M}_{c}$ and $M_{c}$ are trivial (Theorem 2.2).

Received 26 April 2004, accepted in final form 2 July 2004, published online 21 June 2005.

2000 Mathematics Subject Classification 14H60, 14F25, 14F42.

Keywords: irreducible symplectic variety, moduli space, sheaf, K3 surface, desingularization.

Partially supported by KOSEF R01-2003-000-11634-0 and KRF 2003-070-C00001.

This journal is (C) Foundation Compositio Mathematica 2005. 


\section{Symplectic Desingularization OF MODUli SPACES OF SHEAVES}

In particular, we deduce that the stringy Euler number $e_{\mathrm{st}}\left(M_{c}\right)$ must be an integer. Therefore, Theorem 1.2 is a consequence of the following.

Proposition 1.3. The stringy Euler number $e_{\mathrm{st}}\left(M_{2 n}\right)$ is of the form

$$
\frac{n a_{n}}{2 n-3}+\text { integer. }
$$

We prove this proposition in $\S 3$ after a brief review of preliminaries.

One motivation for Question 1.1 is to find a mathematical interpretation of Vafa-Witten's formula [VW94, (4.17)] which says that the 'Euler characteristic' of $M_{2 n}$ is

$$
e^{\mathrm{VW}}\left(M_{2 n}\right)=a_{4 n-3}+\frac{1}{4} a_{n} .
$$

Because $k / 4 \neq l /(2 n-3)$ for $1 \leqslant k \leqslant 3,1 \leqslant l<2 n-3$, we deduce the following from Proposition 1.3.

COROLlary 1.4. The stringy Euler number $e_{\text {st }}\left(M_{2 n}\right)$ is not Vafa-Witten's Euler characteristic $e^{\mathrm{VW}}\left(M_{2 n}\right)$ in general.

Independently, Kaledin and Lehn [KL04a] proved that there is no symplectic desingularization of $M_{2 n}$ for any $n \geqslant 3$ by a very different method.

\section{Preliminaries}

In this section, we recall the definition and basic facts about stringy Euler numbers. The references are [Bat98, DL99].

Let $W$ be a variety with at worst log-terminal singularities, i.e.:

- $W$ is $\mathbb{Q}$-Gorenstein;

- for a resolution of singularities $\rho: V \rightarrow W$ such that the exceptional locus of $\rho$ is a divisor $D$ whose irreducible components $D_{1}, \ldots, D_{r}$ are smooth divisors with only normal crossings, we have

$$
K_{V}=\rho^{*} K_{W}+\sum_{i=1}^{r} a_{i} D_{i}
$$

with $a_{i}>-1$ for all $i$, where $D_{i}$ runs over all irreducible components of $D$. The divisor $\sum_{i=1}^{r} a_{i} D_{i}$ is called the discrepancy divisor.

For each subset $J \subset I=\{1,2, \ldots, r\}$, define $D_{J}=\bigcap_{j \in J} D_{j}, D_{\emptyset}=Y$ and $D_{J}^{0}=D_{J}-\bigcup_{j \in I-J} D_{j}$. Then the stringy $E$-function of $W$ is defined by

$$
E_{\mathrm{st}}(W ; u, v)=\sum_{J \subset I} E\left(D_{J}^{0} ; u, v\right) \prod_{j \in J} \frac{u v-1}{(u v)^{a_{j}+1}-1}
$$

where

$$
E(Z ; u, v)=\sum_{p, q} \sum_{k \geqslant 0}(-1)^{k} h^{p, q}\left(H_{c}^{k}(Z ; \mathbb{C})\right) u^{p} v^{q}
$$

is the Hodge-Deligne polynomial for a variety $Z$. Note that the Hodge-Deligne polynomials have:

- the additive property: $E(Z ; u, v)=E(U ; u, v)+E(Z-U ; u, v)$ if $U$ is a smooth open subvariety of $Z$;

- the multiplicative property: $E(Z ; u, v)=E(B ; u, v) E(F ; u, v)$ if $Z$ is a locally trivial $F$-bundle over $B$. 


\section{Y.-H. KIEM}

Definition 2.1. The stringy Euler number is defined as

$$
e_{\mathrm{st}}(W)=\lim _{u, v \rightarrow 1} E_{\mathrm{st}}(W ; u, v)=\sum_{J \subset I} e\left(D_{J}^{0}\right) \prod_{j \in J} \frac{1}{a_{j}+1}
$$

where $e\left(D_{J}^{0}\right)=E\left(D_{J}^{0} ; 1,1\right)$.

The 'change of variable formula' (Theorem 6.27 in [Bat98], Lemma 3.3 in [DL99]) implies that the function $E_{\mathrm{st}}$ is independent of the choice of a resolution and the following holds.

Theorem 2.2 [Bat98, Theorem 3.12]. Suppose $W$ is a $\mathbb{Q}$-Gorenstein algebraic variety with at worst log-terminal singularities. If $\rho: V \rightarrow W$ is a crepant desingularization (i.e. $\rho^{*} K_{W}=K_{V}$ ) then $E_{\mathrm{st}}(W ; u, v)=E(V ; u, v)$. In particular, $e_{\mathrm{st}}(W)=e(V)$ is an integer.

\section{Proof of Proposition 1.3}

We fix a generic polarization of $X$ as in [Ogr99, p. 50]. The moduli space $M_{2 n}$ has a stratification

$$
M_{2 n}=M_{2 n}^{s} \sqcup(\Sigma-\Omega) \sqcup \Omega
$$

where $M_{2 n}^{s}$ is the locus of stable sheaves and

$$
\Sigma \cong\left(X^{[n]} \times X^{[n]}\right) / \text { involution }
$$

is the locus of sheaves of the form $I_{Z} \oplus I_{Z^{\prime}}\left([Z],\left[Z^{\prime}\right] \in X^{[n]}\right)$ while

$$
\Omega \cong X^{[n]}
$$

is the locus of sheaves $I_{Z} \oplus I_{Z}$. Kirwan's desingularization $\rho: \widehat{M}_{2 n} \rightarrow M_{2 n}$ is obtained by blowing up $M_{c}$ first along the deepest stratum $\Omega$, next along the proper transform of the middle stratum $\Sigma$ and finally along the proper transform of a subvariety $\Delta$ in the exceptional divisor of the first blow-up which is the locus of $\mathbb{Z}_{2}$ quotient singularities [Kir85]. This is indeed a desingularization by [Ogr99, Proposition 1.8.3].

Let $D_{1}=\widehat{\Omega}, D_{2}=\widehat{\Sigma}, D_{3}=\widehat{\Delta}$ be the (proper transforms of the) exceptional divisors of the three blow-ups. Then they are smooth divisors with only normal crossings and the discrepancy divisor of $\rho: \widehat{M}_{2 n} \rightarrow M_{2 n}$ is $[\operatorname{Ogr} 99,(6.1)]$

$$
(6 n-7) D_{1}+(2 n-4) D_{2}+(4 n-6) D_{3} .
$$

Therefore the singularities are terminal for $n \geqslant 2$ and from (2.2) the stringy Euler number of $M_{2 n}$ is given by

$$
\begin{aligned}
e\left(M_{2 n}^{s}\right)+e & \left(D_{1}^{0}\right) \frac{1}{6 n-6}+e\left(D_{2}^{0}\right) \frac{1}{2 n-3}+e\left(D_{3}^{0}\right) \frac{1}{4 n-5}+e\left(D_{12}^{0}\right) \frac{1}{6 n-6} \frac{1}{2 n-3} \\
& +e\left(D_{23}^{0}\right) \frac{1}{2 n-3} \frac{1}{4 n-5}+e\left(D_{13}^{0}\right) \frac{1}{6 n-6} \frac{1}{4 n-5}+e\left(D_{123}^{0}\right) \frac{1}{6 n-6} \frac{1}{2 n-3} \frac{1}{4 n-5} .
\end{aligned}
$$

We need to compute the (virtual) Euler numbers of $D_{J}^{0}$ for $J \subset\{1,2,3\}$. Let $(E, \omega)$ be a symplectic vector space of dimension $c=2 n$. Let $\operatorname{Gr}^{\omega}(k, c)$ be the Grassmannian of $k$-dimensional subspaces of $E$ isotropic with respect to the symplectic form $\omega$ (i.e. the restriction of $\omega$ to the subspace is zero).

Lemma 3.1. For $k \leqslant n$, the Euler number of $\operatorname{Gr}^{\omega}(k, 2 n)$ is $2^{k}\left(\begin{array}{l}n \\ k\end{array}\right)$.

Proof. Consider the incidence variety

$$
\left\{(a, b) \in \operatorname{Gr}^{\omega}(k-1,2 n) \times \operatorname{Gr}^{\omega}(k, 2 n) \mid a \subset b\right\} .
$$




\section{Symplectic Desingularization OF MODUli SPACES OF SHEAVES}

This is a $\mathbb{P}^{2 n-2 k+1}$-bundle over $\operatorname{Gr}^{\omega}(k-1,2 n)$ and a $\mathbb{P}^{k-1}$-bundle over $\operatorname{Gr}^{\omega}(k, 2 n)$. The formula follows from an induction on $k$.

Let $\hat{\mathbb{P}}^{5}$ be the blow-up of $\mathbb{P}^{5}$ (projectivization of the space of $3 \times 3$ symmetric matrices) along $\mathbb{P}^{2}$ (the locus of rank 1 matrices). We have the following from [Ogr99, § 6] and [Ogr97, § 3].

Proposition 3.2.

(1) $D_{1}$ is a $\hat{\mathbb{P}}^{5}$-bundle over a $\operatorname{Gr}^{\omega}(3,2 n)$-bundle over $X^{[n]}$.

(2) $D_{2}^{0}$ is a $\mathbb{P}^{2 n-4}$-bundle over a $\mathbb{P}^{2 n-3}$-bundle over $\left(X^{[n]} \times X^{[n]}-X^{[n]}\right) /$ involution.

(3) $D_{3}$ is a $\mathbb{P}^{2 n-4} \times \mathbb{P}^{2}$-bundle over a $\operatorname{Gr}^{\omega}(2,2 n)$-bundle over $X^{[n]}$.

(4) $D_{1} \cap D_{2}$ is a $\mathbb{P}^{2} \times \mathbb{P}^{2}$-bundle over $\mathrm{Gr}^{\omega}(3,2 n)$-bundle over $X^{[n]}$.

(5) $D_{2} \cap D_{3}$ is a $\mathbb{P}^{2 n-4} \times \mathbb{P}^{1}$-bundle over a $\operatorname{Gr}^{\omega}(2,2 n)$-bundle over $X^{[n]}$.

(6) $D_{1} \cap D_{3}$ is a $\mathbb{P}^{2} \times \mathbb{P}^{2 n-5}$-bundle over a $\operatorname{Gr}^{\omega}(2,2 n)$-bundle over $X^{[n]}$.

(7) $D_{1} \cap D_{2} \cap D_{3}$ is a $\mathbb{P}^{1} \times \mathbb{P}^{2 n-5}$-bundle over a $\operatorname{Gr}^{\omega}(2,2 n)$-bundle over $X^{[n]}$.

For instance, (1) is just Proposition 6.2 of [Ogr99] and (2) is Proposition 3.3.2 of [Ogr97], while (3) is Lemma 3.5.4 in [Ogr97].

From Proposition 3.2 and Lemma 3.1, we have the following by the additive and multiplicative properties of the (virtual) Euler numbers:

$$
\begin{array}{rlrl}
e\left(D_{1}^{0}\right) & =0, & e\left(D_{2}^{0}\right) & =(2 n-3)(2 n-2) \frac{1}{2}\left(a_{n}^{2}-a_{n}\right), \\
e\left(D_{3}^{0}\right) & =2^{2}\left(\begin{array}{l}
n \\
2
\end{array}\right) a_{n}, & e\left(D_{12}^{0}\right) & =3 \cdot 2^{3}\left(\begin{array}{l}
n \\
3
\end{array}\right) a_{n} \\
e\left(D_{23}^{0}\right) & =2 \cdot 2^{2}\left(\begin{array}{l}
n \\
2
\end{array}\right) a_{n}, & e\left(D_{13}^{0}\right) & =(2 n-4) 2^{2}\left(\begin{array}{l}
n \\
2
\end{array}\right) a_{n} \\
e\left(D_{123}^{0}\right) & =2(2 n-4) 2^{2}\left(\begin{array}{l}
n \\
2
\end{array}\right) a_{n} . &
\end{array}
$$

Hence from the formula (3.1), the stringy Euler number of $M_{2 n}$ is given by

$$
e_{\mathrm{st}}\left(M_{2 n}\right)=e\left(M_{2 n}^{s}\right)+(n-1)\left(a_{n}^{2}-a_{n}\right)+n \frac{2 n-2}{2 n-3} a_{n}=\frac{n a_{n}}{2 n-3}+\text { integer }
$$

since $e\left(M_{2 n}^{s}\right)$ is an integer. So we have proved Proposition 1.3.

Remark 3.3. For the moduli space of rank 2 bundles over a smooth projective curve, the stringy E-function and the stringy Euler number are computed in [Kie03] and [KL04b].

\section{ACKNowledgements}

It is my great pleasure to express my gratitude to Professor Jun Li for useful discussions and to Jaeyoo Choy for motivating questions.

\section{REFERENCES}

Bat98 V. Batyrev, Stringy Hodge numbers of varieties with Gorenstein canonical singularities, in Integrable systems and algebraic geometry (Kobe/Kyoto, 1997) (World Scientific, River Edge, NJ, 1998), 1-32.

DL99 J. Denef and F. Loeser, Germs of arcs on singular varieties and motivic integration, Invent. Math. 135 (1999), 201-232.

Kie03 Y.-H. Kiem, The stringy E-function of the moduli space of rank 2 bundles over a Riemann surface of genus 3, Trans. Amer. Math. Soc. 355 (2003), 1843-1856. 


\section{Symplectic Desingularization of MOduli SPACES OF SHEAVES}

Kir85 F. Kirwan, Partial desingularisations of quotients of nonsingular varieties and their Betti numbers, Ann. of Math. (2) 122 (1985), 41-85.

KL04a D. Kaledin and M. Lehn, Local structure of hyperkaehler singularities in O'Grady's examples, Preprint (2004), math.AG/0405575.

KL04b Y.-H. Kiem and J. Li, Desingularizations of the moduli space of rank 2 bundles over a curve, Math. Ann. 330 (2004), 491-518.

Ogr97 K. G. O'Grady, Desingularized moduli spaces of sheaves on a K3, Preprint (1997), alg-geom/9708009.

Ogr99 K. G. O'Grady, Desingularized moduli spaces of sheaves on a K3, J. reine angew. Math. 512 (1999), 49-117.

VW94 C. Vafa and E. Witten, A strong coupling test of S-duality, Nucl. Phys. B 431 (1994), 3-77.

Young-Hoon Kiem kiem@math.snu.ac.kr

Department of Mathematics, Seoul National University, Seoul 151-747, Korea 